\title{
STATIC ANALYSIS OF CIRCULAR CYLINDRICAL SHELL UNDER HYDROSTATIC AND RING FORCES
}

\author{
N. N. Osadebe and A. Adamou, \\ Department of Civil Engineering \\ University of Nigeria, Nsukka.
}

\begin{abstract}
Analysis of circular cylindrical shell under the action of hydrostatic and stiffening ring forces is carried out in this work. The differential equation of equilibrium, similar to that of beam on elastic foundation, was obtained from static principles on the assumptions of P. L. Pasternak. The initial value method was used to solve the obtained fourth order differential equation for both cases of hydrostatic and ring forces. Combined actions of hydrostatic and ring forces were studied as the location of the ring was varied along the height of the reservoir. It appears that the most favourable location for the ring is $2 / 3$ of its height measured from the top. Bending moment, shear force and hoop tension diagrams, essentially necessary for design of the reservoir, were plotted under the action of these forces.
\end{abstract}

Keywords: Static analysis, cylindrical shell, hydrostatic forces, reservoir, initial value.

\section{INTRODUCTION}

The economy or feasibility of many modern constructions necessitates lightweight, thinwalled members. Indeed the objective of structural engineering has always been to lower as much as possible the cost and thus the quantity of construction material without compromising the integrity of the structure. Thin-walled structures, which include both thin plates and thin shells, satisfy the afore-mentioned objective. Shells are for most part the deep-seated structures in manufacturing submarines, missiles, tanks and their roofs, and fluid reservoirs (Golzan et al, 2008). Circular cylindrical shells are used in a large variety of civil engineering structures, e.g. off-shore platforms, chimneys, silos, pipelines, bridge arches or wind turbine towers (Winterstetter et al, 2002). This work is concerned with the analysis of circular cylindrical shell subjected to hydrostatic pressure in combination with the action of local forces induced by stiffening rings (Fig. 1).

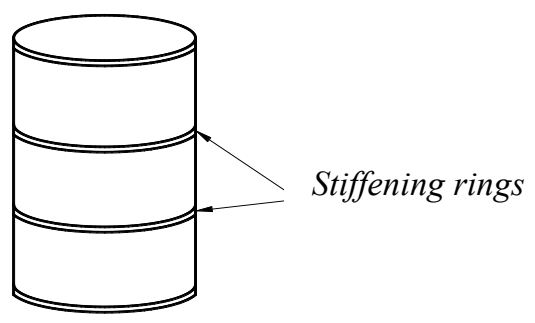

Fig. 1: Stiffened circular cylindrical shell 
In Nigeria, most reservoir constructors introduce stiffening rings with the purpose to overcome bulging due to internal hydrostatic pressure. The location of these rings is generally subject to the very arbitrary appreciation of the constructor. However the introduction of rings so randomly located induces local forces which complicate the stress and strain distribution. Therefore the objective of this study is to give a clear picture of stress and strain distribution in the presence of such induced forces, and to establish the optimum location of the stiffening ring.

The moment theory of shells developed by Aron (Darkov, 1989), and Love (1888) using the analogy of plates due to Kirchoff (1877), in spite of its attractive accuracy, is seldom applied by an average design engineer due to the involved rigorous mathematics. Subsequently, Finsterwalder (1933), Vlasov (1948), and Pasternak (1932), by ignoring the effects of longitudinal bending moment, shear forces and torques arrived at the so-called semi-moment theory. is found to give acceptable results. This method which has been experimentally verified or cylindrical shell whose ratio of length to diameter ranges between 2 to 8 (Timoshenko, 1959).

Pasternak (1932) later showed that when the load on a circular cylindrical shell is axisymmetric, the stresses and strains are functions of only one variable along the axis of the cylinder. The differential equation of equilibrium of the shell in this circumstance reduces to a fourth order linear differential equation equivalent to that of Beam on Elastic Foundation (BEF). This latter equation will be used here on the basis of initial value solution due to Krylov (1931) to study the stresses and strains distribution in cylindrical shell tank subjected to hydrostatic pressure as the location of the stiffening ring varies along the height of the cylinder. The best location of the stiffening ring will be obtained as that location with the least stress and strain on the cylindrical shell.

An abundant literature is available on the use of numerical methods, specially the finite element method, for the analysis of cylindrical shells (Jaroslav, 2005); but recent studies have shown the great dangers of using numerical modelling without a sufficient deep understanding of the effects of choosing different analysis options (Federico et al, 2008). Thus the use of analytical methods, such as the initial value approach, in the analysis of cylindrical shells cannot be overemphasised.

\section{Differential Equation of Equilibrium}

The equations of equilibrium of a cylindrical shell according to the semi-moment theory are (Timoshenko et al, 1959):

$$
\begin{aligned}
& \frac{\partial^{2} u}{\partial x^{2}}+\frac{1-\mu}{2} \cdot \frac{\partial^{2} u}{\partial y^{2}}+\frac{1+\mu}{2} \cdot \frac{\partial^{2} v}{\partial x \partial y}+ \\
& \frac{u}{R} \cdot \frac{\partial w}{\partial x}+\frac{1-\mu^{2}}{E h} \cdot X=0 \\
& \frac{1+\mu}{2} \cdot \frac{\partial^{2} u}{\partial x \partial y}+\frac{1-\mu}{2} \cdot \frac{\partial^{2} v}{\partial x^{2}}+\frac{\partial^{2} v}{\partial y^{2}}+\frac{\partial}{\partial y}\left(\frac{w}{R}\right)+ \\
& \frac{h^{2}}{12 R} \cdot\left[\frac{\partial^{2}}{\partial y^{2}}\left(\frac{v}{R}\right)-\frac{\partial^{3} w}{\partial y^{3}}-\mu \cdot \frac{\partial^{3} w}{\partial x^{2} \partial y}\right]+\frac{1-\mu^{2}}{E h} \cdot Y=0
\end{aligned}
$$

$-\frac{\partial^{4} w}{\partial x^{4}}+\frac{\partial}{\partial y}\left[\frac{u}{R} \cdot \frac{\partial^{2}}{\partial y^{2}}+\frac{\partial^{2}}{\partial y^{2}}\left(\frac{v}{R}\right)\right]-\frac{\partial^{4} w}{\partial y^{4}}$

$2 \mu \cdot \frac{\partial^{4} w}{\partial x^{2} \partial y^{2}}-\frac{12}{R h^{2}}\left[\frac{\partial v}{\partial y}+\frac{w}{R}+\mu \cdot \frac{\partial u}{\partial x}\right]+\frac{12(-\mu)}{E h^{3}} \cdot Z$

where $x, y$ and $z$ axes at a given point $O$ of the middle surface are taken in the directions of the axis of the cylinder, the tangent to the circumference, and the normal to the middle surface of the shell respectively; $X, Y$, and $Z$ are the components of the transverse distributed load in $x$, $y$, and $z$ directions respectively; $u, v$, and $w$ are the displacement components in $x, y$, and $z$ directions respectively;

$h$ is the thickness of the shell;

$E$ is Young modulus of the material;

$R$ is the radius of the cylinder; and $\mu$ is Poisson's ratio. 
On the assumptions that (i) the middle surface of the shell is inextensible in $y$ - direction such that $v=0$, and (ii) the normal force $N_{x}$ acting on the transverse section of the shell is neglected, Pasternak (1932) arrived at the following relations:

$\frac{d u}{d x}=u^{1}=-\mu \frac{w}{R}$

$N_{\varphi}=N=\frac{E h w}{R}$

$S=0$

$M_{x}=M=-D w^{n}$

$M_{\varphi}=\mu M$

Where $\quad w^{n}=\frac{d^{2} w}{d x^{2}} ;$ and

$$
D=\frac{E h^{3}}{12\left(1-\mu^{2}\right)}
$$

$N_{\varphi}$ is the hoop tension;

$S$ is the membrane shearing force; $M_{x}$ is the longitudinal moment; and $M_{\varphi}$ is the transverse moment.

Substituting the above relations into equation [3] and considering the hydrostatic loading, we obtain:

$\frac{d^{4} w}{d x^{4}}+4 \beta w=\frac{\gamma^{x}}{D}$

where: $\beta^{4}=\frac{3\left(1-\mu^{2}\right)}{R^{2} h^{2}}$

Equation (9) is due to Pasternak (1932) and is only applicable to cylindrical shell subject to axi-symmetric loading i.e. stresses and strains are constant along the circumferential section.

\section{The Initial Value Solution}

Let $\mathrm{Z}=\beta x$

Differentiating the above with respect to $x$ gives: $\frac{d z}{d x}=\beta$

In general:

$\frac{d^{n}}{d x^{n}}=\frac{d^{n}}{d z^{n}}\left(\frac{d z}{d x}\right)^{n}=\beta^{n} \frac{d^{n}}{d z^{n}}$

Thus, equation (9) becomes:

$\frac{d^{4} w}{d z^{4}}+4 w(z)=\left(\frac{4 \gamma R^{2}}{E h \beta} z\right)$

\section{Initial Value Homogeneous Solution}

The homogeneous solution can be put in the form:

$w_{h}(z)=\sum_{k=1}^{4} c_{k} Y_{k}(z)$

Where:

$$
\begin{aligned}
\mathrm{Y}_{\mathrm{k}}(\mathrm{z})= & \mathrm{a}_{\mathrm{k}} \cosh z \cos z+b_{k} \cosh \mathrm{z} \sin z+ \\
& c_{k} \sinh z \cos z+d_{k} \sinh z \sin z
\end{aligned}
$$

The constants $a_{k}, b_{k}, c_{k}, d_{k}(k=1,2,3,4), 16$ in number are obtained by differentiating eqn (12) with respect to $z$ till the fourth order and making use of the supplementary initial conditions which are given as: (Krylov, 1931)

$$
\begin{array}{llll}
\mathrm{Y}_{1}(0)=1 & \mathrm{Y}_{1}{ }^{\mathrm{i}}(0)=0 & \mathrm{Y}_{1}{ }^{\mathrm{ii}}(0)=0 \\
\mathrm{Y}_{1}{ }^{i i i}(0)=0 & \mathrm{Y}_{2}(0)=0 & \mathrm{Y}_{2}{ }^{\mathrm{i}}(0)=1 \\
\mathrm{Y}_{2}{ }^{\text {ii }}(0)=0 & \mathrm{Y}_{2}{ }^{\mathrm{iii}}(0)=0 & \mathrm{Y}_{3}(0)=0 \\
\mathrm{Y}_{3}{ }^{\mathrm{i}}(0)=0 & \mathrm{Y}_{3}{ }^{\mathrm{ii}}(0)=1 & \mathrm{Y}_{3}{ }^{\mathrm{iii}}(0)=0 \\
\mathrm{Y}_{4}{ }{ }^{(}(0)=0 & \mathrm{Y}_{4}{ }^{\mathrm{i}}(0)=0 & \mathrm{Y}_{4}{ }^{\mathrm{ii}}(0)=0 \\
\mathrm{Y}_{4}{ }^{\mathrm{iii}}(0)=1 & &
\end{array}
$$

We therefore obtain:

$Y_{1}(z)=\phi_{1}(z)$

$Y_{2}(z)=\frac{1}{2}\left[\phi_{2}(z)+\varphi_{3}(z)\right]$

$Y_{3}(z)=\frac{1}{2} \phi_{4}(z)$

$Y_{4}=\frac{1}{4}\left[\phi_{2}(z)-\varphi_{3}(z)\right]$ 
where:

$\varphi_{1}(\mathrm{z})=\cosh \mathrm{z} \cos \mathrm{z}$

$\varphi_{2}(z)=\cosh z \sin z$

$\varphi_{3}(z)=\sinh z \cos z$

$\varphi_{4}(z)=\sinh z \sin z$

It can be seen that $Y_{k}, k=1,2,3,4$ are four independent expressions. The homogeneous solution will thus be written as:

$w_{h}(z)=c_{1} Y_{1}(z)+c_{2} Y_{2}(z)+c_{3} Y_{3}(z)+c_{4} Y_{4}(z)$

The arbitrary constants $c_{1}, c_{2}, c_{3}$, and $c_{4}$ are to be determined using the initial conditions. The derivatives of the functions $Y_{k}$, essential for the determination of the other stresses and strains, are tabulated [Table 1].

Table 1: $Y_{k}$ functions and their derivatives

\begin{tabular}{ccccc}
\hline & $\mathrm{Y}^{\mathrm{I}}$ & $\mathrm{Y}^{\mathrm{ii}}$ & $\mathrm{Y}^{\mathrm{iii}}$ & $\mathrm{Y}^{\mathrm{iv}}$ \\
\hline $\mathrm{Y}_{1}$ & $-4 \mathrm{Y}_{4}$ & $-4 \mathrm{Y}_{3}$ & $-4 \mathrm{Y}_{2}$ & $-4 \mathrm{Y}_{1}$ \\
$\mathrm{Y}_{2}$ & $\mathrm{Y}_{1}$ & $-4 \mathrm{Y}_{4}$ & $-4 \mathrm{Y}_{3}$ & $-4 \mathrm{Y}_{2}$ \\
$\mathrm{Y}_{3}$ & $\mathrm{Y}_{2}$ & $\mathrm{Y}_{1}$ & $-4 \mathrm{Y}_{4}$ & $-4 \mathrm{Y}_{3}$ \\
$\mathrm{Y}_{4}$ & $\mathrm{Y}_{3}$ & $\mathrm{Y}_{2}$ & $\mathrm{Y}_{1}$ & $-4 \mathrm{Y}_{4}$ \\
\hline
\end{tabular}

The relationships between the deflection $(w(z))$ and the rotation $(q(z))$, moment $(M(z))$, shear force $(Q(z))$ and hoop tension $(N(z))$ are derived in the following manner.

Owing to:

$$
\begin{aligned}
& \frac{d^{n} w}{d x^{n}}=\beta^{n} \frac{d^{n} w}{d z^{n}}, \text { we obtain: } \\
& \theta_{h}(z)=\frac{d w_{h}}{d x}=\beta w_{h}^{1}(z)=-4 \beta c_{1} Y_{4}(z)+ \\
& \beta c_{2} Y_{1}(z)+\beta c_{3} Y_{2}(z)+\beta c_{4} Y_{3}(z)
\end{aligned}
$$

$$
\begin{aligned}
& M_{h}(z)=-D \frac{d^{2} w_{h}}{d x^{2}}=-D \beta^{2} w_{h}^{i i}(z)=\frac{E h}{4 \beta^{2} R^{2}} * \\
& \quad 4 c_{1} Y_{3}(z)+4 c_{2} Y_{4}(z)-c_{3} Y_{1}(z)-c_{4} Y_{2}(z)- \\
& Q_{h}(z)=\frac{d M}{d x}=-D \beta^{3} w_{h}^{i i i}=\frac{E h}{4 \beta R^{2}} * \\
& \begin{array}{|l}
c_{1} Y_{2} \\
(z)+4 c_{2} Y_{3}(z)+4 c_{3} Y_{4}(z)-c_{4} Y_{1}(z)
\end{array} \\
& N_{h}(z)=\frac{E h}{R} w_{h}(z)=\frac{E h}{R} * \\
& \mathbf{E}_{1} Y_{1}(z)+c_{2} Y_{2}(z)+c_{3} Y_{3}(z)+c_{4} Y_{4}(z)
\end{aligned}
$$

where the subscript $h$ stands for "homogeneous".

Taking $W_{o}, q_{o}, M_{o}$, and $Q_{o}$ as initial value of $W_{h}$ (z), $q_{h}(z), M_{h}(z)$ and $Q_{h}(z)$ respectively, the arbitrary constants $c_{k}$ are found by making use of the initial supplementary conditions:

$c_{2}=\frac{\theta_{0}}{\beta}$

$c_{3}=-\frac{4 \beta^{2} R^{2}}{E h} M_{0}$

$c_{4}=-\frac{4 \beta R^{2}}{E h} Q_{0}$

$c_{1}=w_{0}$

Substituting for $c_{1}, c_{2}, c_{3}$, and $c_{4}$ in eqns (22) through (21) we obtain:

$w_{h}(\xi)=Y_{1}(z) v_{0}+\frac{Y_{2}(z)}{\beta} \theta-$
$\frac{4 \beta^{2} R^{2}}{E h} Y_{3}(\xi) M_{0}-\frac{4 \beta R^{2}}{E h} Y_{4}(\xi) \boldsymbol{Q}_{0}$

$\theta_{h}(z)=-4 \beta Y_{4}(z) v_{0}+Y_{1}(z)_{0}-$

$\frac{4 \beta^{3} R^{2}}{E h} Y_{2}(2) M_{0}-\frac{4 \beta^{2} R^{2}}{E h} Y_{3}(\boldsymbol{)})$

$M_{h}(\varepsilon)=\frac{E h}{\beta^{2} R^{2}} Y_{3}(\varepsilon) v_{0}+$

$\frac{E h}{\beta^{3} R^{2}} Y_{4}\left(\xi \varnothing_{0}+Y_{1}(z) M_{0}+\frac{Y_{2}(z)}{\beta} \cdot Q_{0}\right.$ 
$Q_{h}(z)=\frac{E h}{\beta R^{2}} Y_{2}(z) v_{0}+$

$\frac{E h}{\beta^{2} R^{2}} Y_{3}(\beta) B Y_{4}(2) Y_{0}+Y_{1}(\varepsilon) Q_{0}$

$\left.N_{h}(z)=\frac{E h}{R} Y_{1}(z) v_{0}+\frac{E h}{\beta R} Y_{2}(z)\right)_{0}-$

$4 \beta^{2} R Y_{3}(z) M_{0}-4 \beta R Y_{4}(z) Q_{0}$

Initial Value Particular Solution for the Hydrostatic Pressure

To find the particular solution we proceed to an origin transformation (Fig. 2). The origin that was previously at point A (top of the tank) is shifted to point B along the height of the tank, introducing thus a new variable $\mathrm{x}$ ' (at the location of an arbitrary point $\mathrm{C}$ along the height of the tank). Let $d x$ ' be an infinitesimal distance taken from point B. Due to the hydrostatic pressure acting on the vertical side of the tank, a differential (point) load dQ will result at B. Qo is the (initial) shear force acting at A. X stands for the coordinate of the arbitrary point $\mathrm{C}$ when the origin is taken at $\mathrm{A}$. $\mathrm{L}$ is the height of the tank

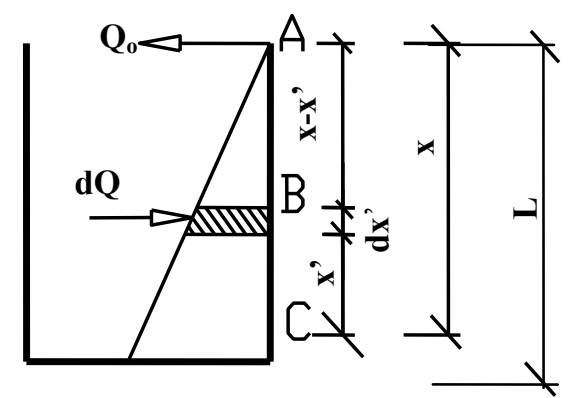

Fig. 2: Hydrostatic loading and origin transformation

$z^{\prime}=\beta x^{\prime} \Rightarrow d x^{\prime}=\frac{d z^{\prime}}{\beta}$

The distributed load at the new origin $B$ is given by:

$q\left(z^{\prime}\right)=\frac{z-z^{\prime}}{\beta} \gamma$
Therefore the elemental force can be expressed as:

$d Q=q\left(z^{\prime}\right) \frac{d z^{\prime}}{\beta}=\frac{\gamma\left(z-z^{\prime}\right)}{\beta^{2}} d z^{\prime}$

With the shift of origin the initial values become:

$Q_{o}=-\mathrm{d} Q$ and $M_{o}=\theta_{o}=w_{o}=0$

Using these values in the expressions of the homogeneous solution, the elemental expressions for the particular solution are obtained:

$$
\begin{aligned}
& d w=\frac{4 \gamma R^{2}}{E h \beta}\left(z-z^{\prime}\right) Y_{4}\left(z^{\prime}\right) d z^{\prime} \\
& d N_{p}=\frac{\gamma}{\beta^{3}}\left(z-z^{\prime}\right)_{2}\left(z^{\prime}\right) d z^{\prime} \\
& d \theta_{p}=\frac{4 \gamma R^{2}}{E h}\left(z-z^{\prime}\right)_{3}\left(z^{\prime}\right) d z^{\prime} \\
& d Q_{p}=-\frac{\gamma}{\beta^{2}}\left(z-z^{\prime}\right)_{1}\left(z^{\prime}\right) d z^{\prime} \\
& d N_{p}=\frac{4 \gamma R}{\beta}\left(z-z^{\prime}\right)^{\prime} Y_{4}\left(z^{\prime}\right) d z^{\prime}
\end{aligned}
$$

where the subscript $p$ denotes "particular".

Integrating the above expressions gives the actual particular solution:

$$
\begin{aligned}
& w_{p}=\int_{0}^{z} d w_{p}=\frac{\gamma R^{2}}{E h \beta}\left[z-Y_{2}(z)\right] \\
& \theta_{p}=\int_{0}^{z} d \theta_{p}=\frac{R^{2} \gamma}{E h}\left[1-Y_{1}(z)\right] \\
& M_{p}=\int_{0}^{z} d M_{P}-\frac{\gamma}{\beta^{3}} Y_{4}(z)
\end{aligned}
$$

$Q_{p}=\int_{0}^{z} d Q_{p}=-\frac{\gamma}{\beta^{2}} Y_{3}(z)$

$N_{p}=\int_{0}^{z} d N_{p} \frac{\gamma R}{\beta}\left[z-Y_{2}(z)\right]$ 
The Initial Value General Solution for the Hydrostatic Pressure

The general solution is obtained by adding homogeneous and particular solutions:

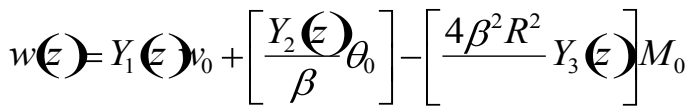

$$
\begin{aligned}
& \left.-\left[\frac{4 \beta R^{2}}{E h} Y_{4}(z)\right] Q_{0}+\frac{\gamma R^{2}}{E h \beta} \bar{z}-Y_{2}(z)\right] \\
& \theta(z)=-4 \beta Y_{4}(z) y_{0}+Y_{1}(z) y_{0}-\left[\frac{4 \beta^{3} R^{2}}{E h} Y_{2}(z)\right] M_{0} \\
& -\left[\frac{4 \beta^{2} R^{2}}{E h} Y_{3}(z)\right] Q_{0}+\frac{R^{2} \gamma}{E h}\left[1-Y_{1}(z)\right. \\
& M(\xi)=\left[\frac{E h}{\beta^{2} R^{2}} Y_{3}(\xi)\right] w_{0}+\left[\frac{E h}{\beta^{3} R^{2}} Y_{4}(\xi)\right] \theta_{0}+Y_{1}(\xi) M_{0} \\
& +\frac{Y_{1}(\xi)}{\beta} Q_{0}-\frac{\gamma}{\beta^{3}} Y_{4}\left(\xi^{\prime}\right. \\
& Q(z)=\left[\frac{E h}{\beta R^{2}} Y_{2}(\varepsilon)\right] w_{0}+\left[\frac{E h}{\beta^{2} R^{2}} Y_{3}(\varepsilon)\right] \theta_{0}-4 \beta Y_{4}(\varepsilon) M_{0} \\
& +Y_{1}\left(z X_{0}-\frac{\gamma}{\beta^{3}} Y_{3}(z)\right. \\
& N(\boldsymbol{\xi})=\left[\frac{E h}{R} Y_{1}()\right] w_{0}+\left[\frac{E h}{\beta R} Y_{2}()\right] \theta_{0}-\left[1 \beta^{2} R Y_{3}\left(M_{0}\right]\right. \\
& -\left[\mathbf{1} \beta R Y_{4}(\boldsymbol{\xi}) \mathbb{\mathbb { P }}_{0}+\frac{\gamma R}{\beta} \mathbf{E}-Y_{2}(\boldsymbol{\xi})\right.
\end{aligned}
$$

\section{Analysis of Radial Ring Force by Displace-} ment Approach

Fig. 3(a) shows the behaviour of a reservoir under hydrostatic pressure: the deflection curve is outward. In order to reduce the effect of the outward deflection, a ring is introduced at a distance $\mathrm{c}$ from the top end. It produces an inward deflection (Fig. 3(b)). One can easily see that the deflection due to hydrostatic pressure and that caused by the ring are opposite one another.

The effects of the hydrostatic and ring forces are first analysed separately and then added to get the combined action. The hydrostatic hav-
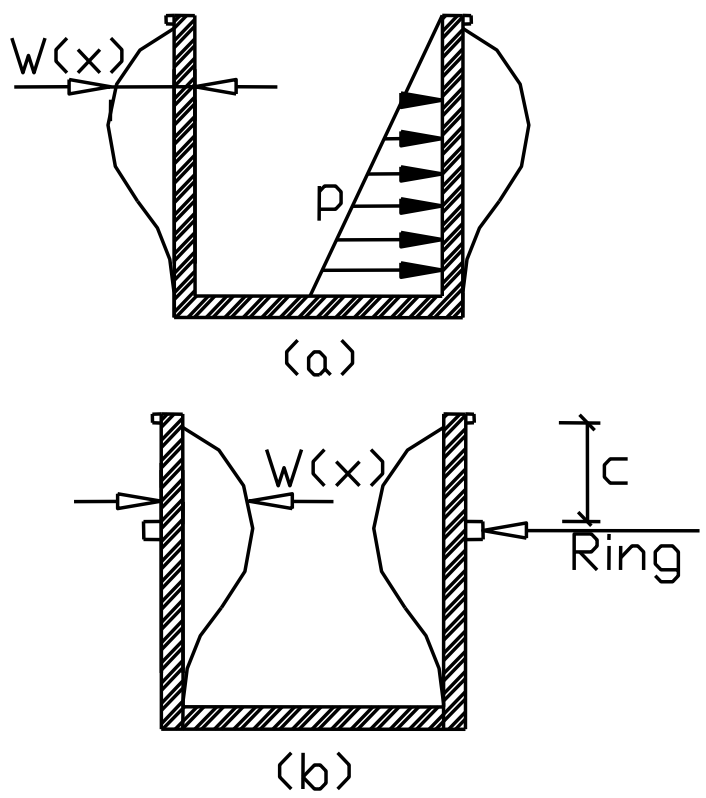

Fig. 3: Deflections due to (a) hydrostatic and (b) ring forces

ing been handled in the previous section, the ring force effect is analysed by displacement approach. The difference between the hydrostatic and ring cases lies only in the particular solution which depends on the loading conditions.

It should be noted that the ring's width is assumed to be negligible compared to the height $L$. It is also supposed that the top end of the tank is supported with an elastic ring of a negligible rigidity against out of plane rotation while the bottom is rigidity fixed to the ground.

It follows that:

$\left.\begin{array}{l}w_{0}=M_{0}=0 \\ w(\beta L)=\theta(\beta L)=0\end{array}\right\}$

The Particular Solution for the Ring Force An origin transformation is made in order to formulate the particular solutions. The new origin is taken at the distance $c$ from the top end. Let the displacement induced by the ring be $\Delta \mathrm{w}$. 
The initial values therefore become:

$W_{o}=-\Delta w$ and $M_{o}=Q_{o}=\theta_{o}$

The variable becomes $z-\beta c$.

Substitution of these into the homogeneous solution gives the particular solution for the ring case:

$w_{p}(z)=-\Delta w Y_{1}(z-t)$

$Q_{p}(z)=-\frac{E h}{\beta R^{2}} \Delta w Y_{2}(z-t)$

$M_{p}(z)=-\frac{E h}{\beta^{2} R^{2}} \Delta w Y_{3}(z-t)$

$Q_{p}(z)=-\frac{E h}{\beta R^{2}} \Delta w Y_{2}(z-t)$

$N_{p}(z)=-\frac{E h}{R} \Delta w Y_{1}(a-t)$

where $t=\beta c$

\section{The General Solution for the Ring Force}

The general solution is obtained by addition of the homogeneous solution and ring force particular solutions and considering the boundary conditions $\left(M_{o}=w_{o}=0\right)$.

Note that the particular solution terms are only added for $z-t \geq 0$.

$w_{r}(z)=\frac{Y_{2}\left(z \phi_{0}\right.}{\beta}-\frac{4 \beta R^{2}}{E h} Y_{4}\left(z \chi_{0}-\Delta w Y_{1}(z-t)\right.$

$\theta_{r}(\varepsilon)=Y_{1}(\varepsilon)_{0}-\frac{4 \beta^{2} R^{2}}{E h} Y_{3}\left(\varepsilon X_{0}+4 \beta \Delta w Y_{4}(z-t)\right.$

$M_{r}(\boldsymbol{\xi}) \frac{E h}{\beta^{3} R^{3}} Y_{4}\left(y_{0}+\frac{Y_{2}(\xi)}{\beta} Q_{0}-\frac{E h}{\beta^{2} R^{2}} \Delta w Y_{3}(-t)\right.$

$Q_{r}(\varepsilon)=\frac{E h}{\beta^{2} R^{2}} Y_{3}\left(\varepsilon \varnothing_{0}+Y_{1}\left(\varepsilon \chi_{0}-\frac{E h}{\beta R^{2}} \Delta w Y_{2}(\varepsilon-t)\right.\right.$

$N_{r}(z)=\frac{E h}{\beta R} Y_{2}(z)_{0}-4 \beta R Y_{4}\left(z \chi_{0}-\frac{E h}{R} \Delta w Y_{1}(\varepsilon-t)\right.$

where the subscript $r$ stands for "ring".

\section{Numerical Studies}

A concrete circular cylindrical water tank with real life dimensions is adopted for numerical purposes:
Radius of reservoir, $R=10 \mathrm{~m}$

Height of reservoir, $L=8 \mathrm{~m}$

Thickness of reservoir, $h=0.2 \mathrm{~m}$

Unit weight of water, $g=9.81 \mathrm{KN} / \mathrm{m}^{3}$

Young's Modulus of Concrete, $E=25 \times 10^{6} \mathrm{KN}$

$\mathrm{m}^{2}$

Poisson's ratio, $m=0.2$

Investigations are carried out for different locations of the ring along the height of the reservoir (2L/3, $L / 2$ and $L / 3$ measured from the top).

The ring is assumed to induce an inward deflection equal in absolute value to half of the outward deflection due to the hydrostatic pressure at its location.

For the different ring locations the combined deflection curve, bending moment, shear force and hoop tension diagrams are plotted.

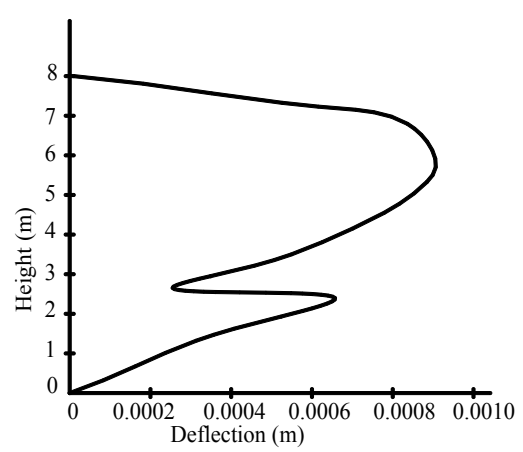

Fig. 4(a): Deflection curve for the ring located at $\mathrm{L} / 3$

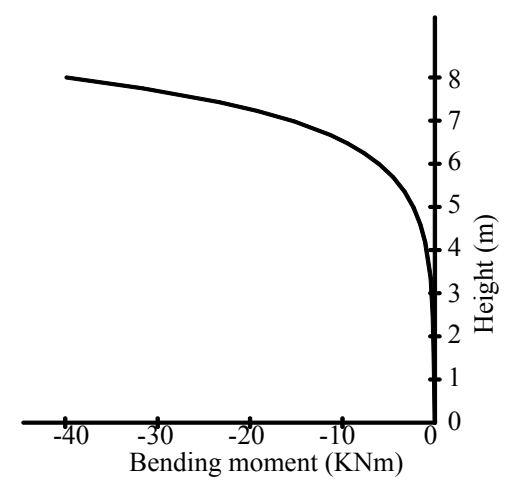

Fig. 4(b): Bending moment diagram for the ring located at $\mathrm{L} / 3$ 


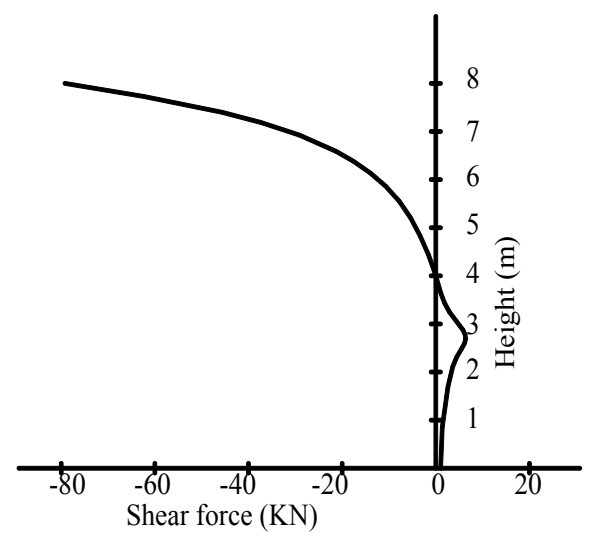

Fig. 4(c): Shear force diagram for the ring located at $\mathrm{L} / 3$

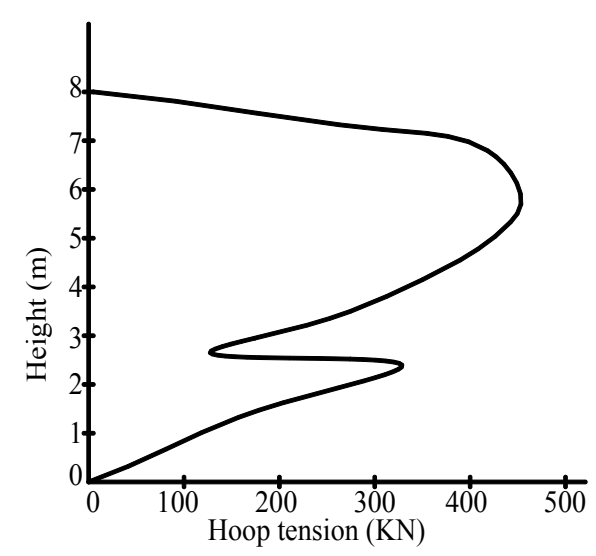

Fig. 4(d): Hoop tension diagram for the ring located at $\mathrm{L} / 3$

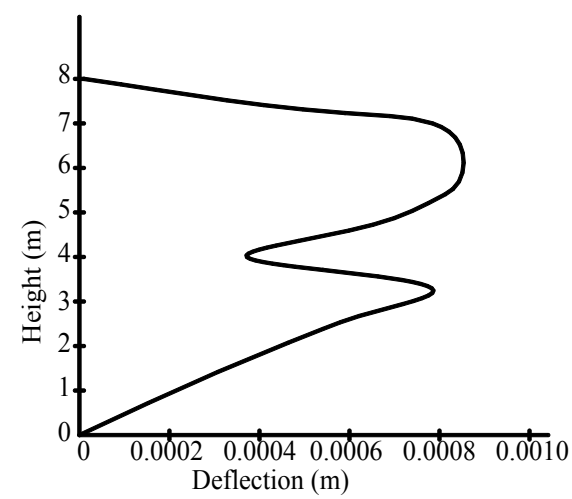

Fig. 5(a): Deflection curve for the ring located at $\mathrm{L} / 2$

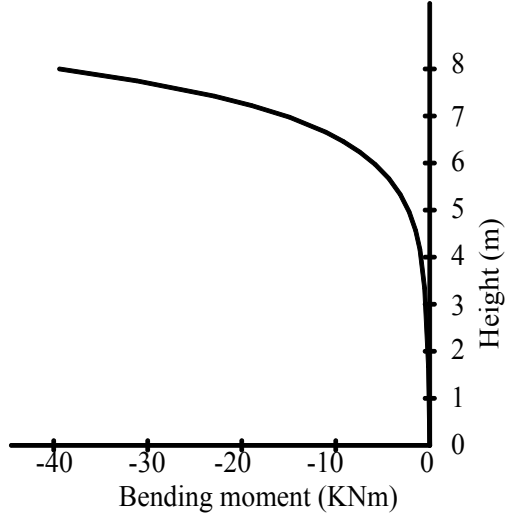

Fig. 5(b): Bending moment diagram for the ring located at $\mathrm{L} / 2$

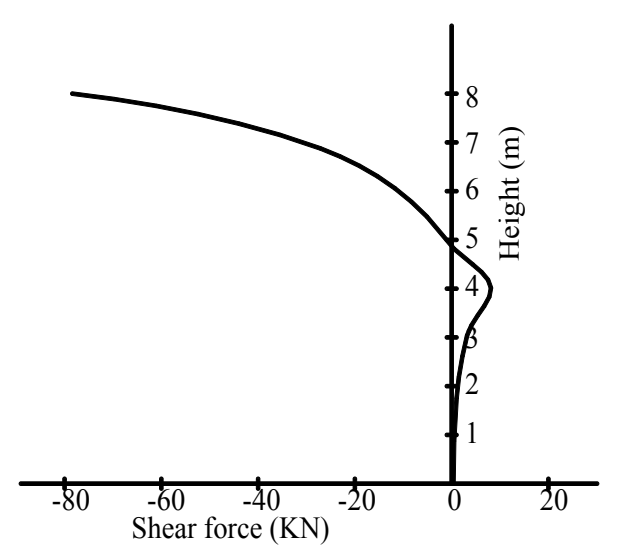

Fig. 5(c): Shear force diagram for the ring located at $\mathrm{L} / 2$

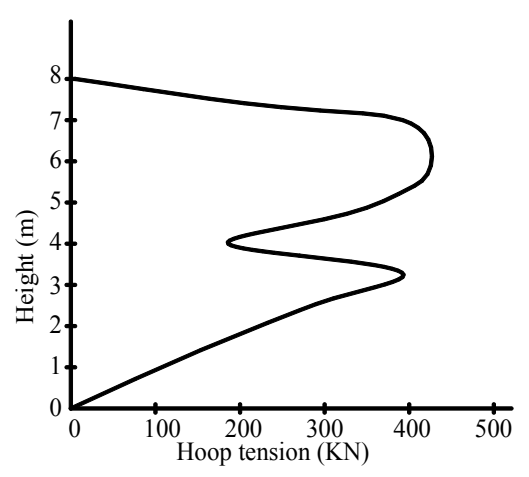

Fig. 5(d): Hoop tension diagram for the ring located at $\mathrm{L} / 2$ 


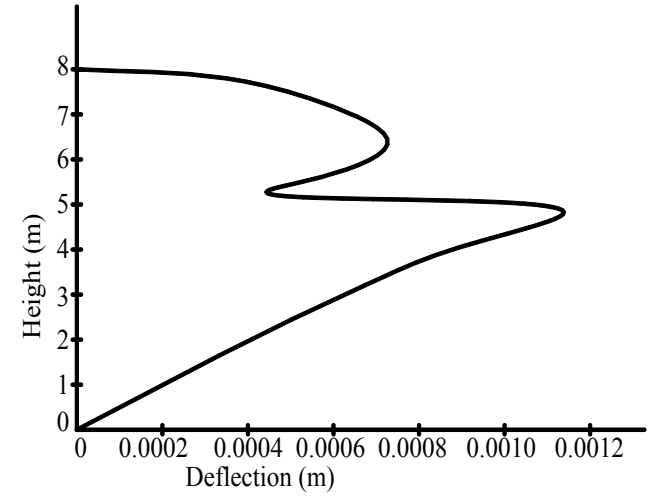

Fig. 6(a): Deflection curve for the ring located at $2 \mathrm{~L} / 3$

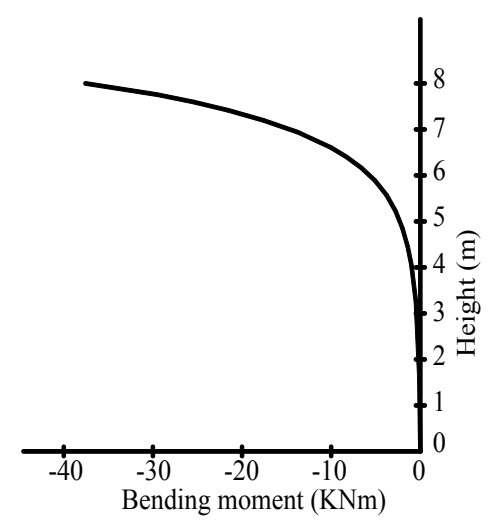

Fig. 6(b): Bending moment diagram for the ring located at $2 \mathrm{~L} / 3$

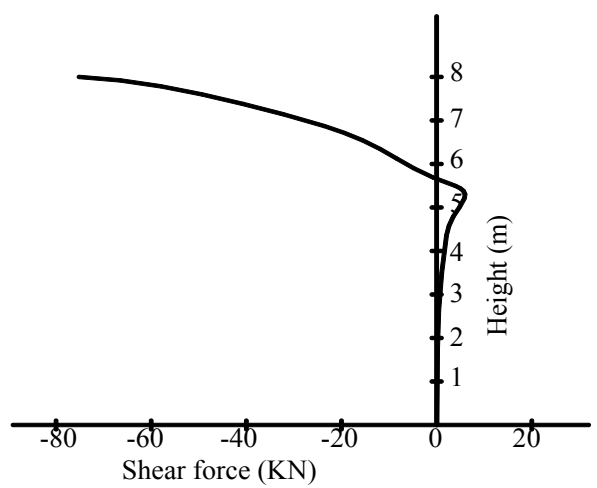

Fig. 6(c): Shear force diagram for the ring located at $2 \mathrm{~L} / 3$

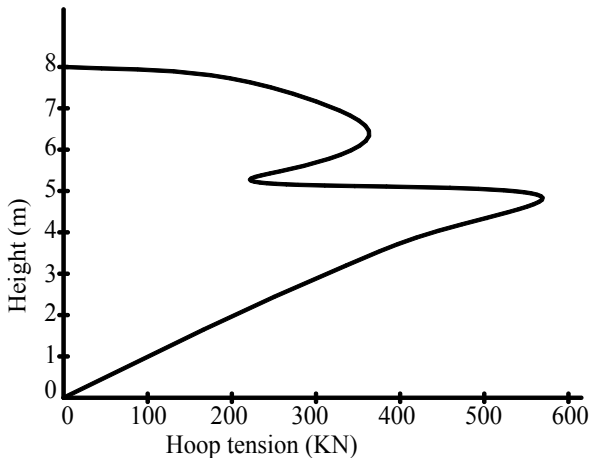

Fig. 6(d): Hoop tension diagram for the ring located at $2 \mathrm{~L} / 3$

\section{DISCUSSION}

Direct Deflection

For the combined action of hydrostatic and ring forces, the maximum deflection would be $0.904 \mathrm{~mm}$ (at $x=5.6 \mathrm{~m}$ ), $0.850 \mathrm{~mm}$ (at $x=6.4 \mathrm{~m}$ ) and $0.727 \mathrm{~mm}$ (at $x=6.4 \mathrm{~m}$ ) when the ring is located respectively at $L / 3, L / 2$ and $2 L / 3$ measured from the top [Fig. 4(a), 5(a), 6(a)]. There is a sudden fall of the deflection at the ring location.

\section{Bending Moment}

For the hydrostatic loading, the bending moment varies from zero, at the top, to $39.969 \mathrm{KNm}$, at the bottom, describing a concave parabolic-like curve due to the cantilever action. With the ring action, the maximum bending moment is reduced to $39.847,39.385$, and $37.563 \mathrm{KNm}$ when the ring is located at $L / 3, L / 2$ and $2 L / 3$ respectively [Fig. 4 (b), 5(b), 6(b)].

\section{Shear Force}

When the hydrostatic pressure is acting alone, the shear force varies from $-0.098 \mathrm{KN}$, at the top, to $-79.417 \mathrm{KN}$, at the bottom. It becomes $79.214,-78.405$, and -75.166 as the ring is placed at $L / 3, L / 2$ and $2 L / 3$. A positive peak is reached at the ring location: $6.349,8.131$, and $5.905 \mathrm{KN}$ for the three ring locations respectively [Fig. 4(c), 5(c), 6(c)]. 


\section{Hoop Tension}

As the hoop tension is directly proportional to the deflection, the graphs have the same shape as those obtained for direct deflection [Fig. 4 (d), 5(d), 6(d)].

\section{CONCLUSION}

The initial value method is more convenient for analysis of ring force compared to other methods such as the classical method, which may involve a more complex procedure to generate the particular integral. The displacement approach shows that the ring can be considered as a way to control the deflection along the height of the reservoir. The introduction of stiffening ring reduces the deflection, but alters the strain distribution at its location. This may explain the busting at ring vicinity occurring for many cases of tank with stiffening ring subjected to hydrostatic pressure. As shown by the plotted graphs, the most favourable location for the ring appears to be at $2 / 3$ of the height of the reservoir measured from its top.

Large ring width could be suggested in order to avoid subsequent busting at the ring location. Further studies could be undertaken in the following areas:

i) analysis of ring force by the classical method;

ii) comparison between displacement and force approaches as applied in the initial value method for analysis of ring force;

iii) behaviour of tanks subject to rings of large width.

\section{REFERENCES}

Darkov A. (1989), Structural Mechanics, $4^{\text {th }}$ Edition, Mir Publishers, Moscow.

Federico Guarracino, and Alastair Walker (2008), Some comments on the numerical analysis of plates and thin-walled structures, Thin-Walled Struct., 46: 975-980.

Finsterwalder U. (1933), Die Theorie der Zylindrischen Schalengewolbe SystemZeiss-Dywidag und ihre Anwendung ouf die Gross-markthalle, Budapest, Int. Vereinig Brucken-u. Hochbau, Abn. 1; Ing. Arch.

Golzan B. S., and Showkati H. (2008), Buckling of thin-walled conical shells under uniform external pressure, Thin-Walled Struct. 46: 516-529.

Jaroslav Mackerle (2005), Finite elements in the analysis of pressure vessels and piping, an addendum: A bibliography (20012004), International Journal of Pressure Vessels and Piping 82: 571-592.

Kirchhoff G. R. (1877), Sungenüber Mathematishe Physik, Mechanik, p. 450

Krylov A. N. (1931), On the Analysis of Beams on Elastic Foundation, Publication of Acad. of Sci., USSR (in Russian).

Love A. E. H. (1888), Philosophical Transactions, Royal Society, 179 (A).

Pasternak P. L. (1932), Practical Calculations for Folds and Cylindrical Shells Taking Bending Moments into Account, Stroitelny byulleten, 9, 10 .

Timoshenko S.P., and Woinowsky-Krieger S. (1959), Theory of Plates and Shells, $2^{\text {nd }}$ Edition, McGRAW-HILL, New York.

Vlasov V.Z. (1949), A General Theory of Shells, Mir Publishers, Moscow.

Winterstetter Th. A., and Schmidt H. (2002), Stability of circular cylindrical steel shells under combined loading, Thin-Walled Struct. 40: 893-909 